\title{
O óleo de girassol como agente de glazeamento em massas para a produção de pães tipo forma
}

\author{
R. A. ZAMBELLI ${ }^{1}$, L. F. L. HERCULANO ${ }^{1}$, D. L. BRASIL ${ }^{1}$, S. C. P. MELO ${ }^{1}$, E. R. \\ PONTES $^{1}$, L. I. F. PINTO ${ }^{1}$ e D. F. PONTES ${ }^{1}$. \\ ${ }^{1}$ Universidade Federal do Ceará, Departamento de Tecnologia de Alimentos. \\ E-mail para contato: Zambelli@alu.ufc.br
}

\begin{abstract}
RESUMO - O estudo teve o objetivo de avaliar a ação do óleo de girassol como agente de glazeamento em massas para a produção de pães. Para o estudo, utilizou-se soluções óleo de girassol/água em concentrações variando de 0 a $50 \mathrm{~mL}$, sendo estas, as variáveis independentes. O efeito do glazeamento foi observado no fator de expansão, volume produzido e específico, densidade e índice de expansão dos pães, foi utilizado o utilizando o delineamento composto central rotacional e a análise feita por metodologia de superfície de resposta. A faixa ótima do fator de expansão consistiu para valores superiores entre a 2,0 cm³ , com adição de 10 a $30 \mathrm{~mL}$ de óleo e 0 a $10 \mathrm{~mL}$ de água. Para o volume produzido, a zona de otimização correspondeu a valores superiores a $20 \mathrm{~mL}$. A densidade obteve valores abaixo de $0,3 \mathrm{~g} / \mathrm{mL}$ na otimização e o índice de expansão com valores maiores que 1,40. Pode-se concluir que soluções contendo até $25 \mathrm{~mL}$ de água $30 \mathrm{~mL}$ de óleo de girassol promoveram melhoria dos parâmetros físicos de qualidade.
\end{abstract}

\subsection{INTRODUÇÃO}

Os produtos de panificação começam a sofrer deterioração da sua qualidade logo após o seu forneamento e durante o armazenamento por causa do endurecimento e de contaminação microbiana. O processo de endurecimento torna o miolo mais rígido, seco e quebradiço, enquanto a crosta setorna macia e com aparência semelhante à couro (Cauvain, 2009). O endurecimento do pão pode ser dividido em duas partes: o endurecimento do miolo e da crosta, de acordo com Primo-Martin et al. (2006) o endurecimento do miolo é de maior preocupação industrial pois está relacionado diretamente com a aceitabilidade do produto por parte do consumidor.

Uma das formas de reduzir à perca de qualidade dos produtos de panificação durante o armazenamento é a utilização da técnica de glazeamento, as soluções, de acordo com Chin et al. (2010), podem ser usadas para produzir diferentes efeitos, fornecendo brilho, melhorando a aparência, retenção de umidade, além de ajudar a prolongar a vida útil dos produtos.

Casper et al. (2007) afirmam que o glazeamento em produtos de panificação possui propriedade de barreira à umidade e retenção de água, que permite a retenção da umidade durante a etapa final de forneamento, o que faz com que colabore com a expansão da massa.

O estudo tem como objetivo estudar o uso de diferentes solições contendo água e óleo de girassol como agente de glazeamento em pães tipo forma e verificar o efeito sobre as características físicas de qualidade dos produtos. 


\subsection{METODOLOGIA}

\subsection{Delineamento Experimental}

O desenvolvimento das formulações de pães tipo forma foi realizado a partir de uma formulação padrão, cuja composição é: $100 \%$ de farinha de trigo; $55-60 \%$ de água, $10 \%$ de Gordura Vegetal Hidrogenada; 5\% de açúcar, 3,3\% de fermento biológico e 2\% de sal.

Na tabela 1 são apresentados as faixas de valores codificados e reais utilizados para os ensaios e na tabela 2 é apresentada a matriz do planejamento.

Tabela 1 - Variáveis e níveis do planejamento experimental completo $2^{2}$

\begin{tabular}{cccccc}
\hline & \multicolumn{4}{c}{ Níveis codificados e reais das variáveis independentes } \\
\cline { 2 - 6 } Variáveis Independentes & $\mathbf{- \alpha}=\mathbf{- 1 , 4 1}$ & $\mathbf{- 1}$ & $\mathbf{0}$ & $\mathbf{+ 1}$ & $\mathbf{+ \alpha}=\mathbf{+ 1 , 4 1}$ \\
Óleo de Girassol $(\mathbf{m L})$ & 0 & 12,5 & 25 & 37,5 & 50 \\
Água $(\mathbf{m L})$ & 0 & 12,5 & 25 & 37,5 & 50 \\
\hline
\end{tabular}

*Em relação a farinha de trigo (base 100).

Para o desenvolvimento das formulações de pães tipo forma incorporadas com diferentes quantidades de óleo de girassol e água foi utilizado o Delineamento Composto Central Rotacional (DCCR). Foi aplicado um planejamento fatorial $2^{2}$ completo, totalizando 11 ensaios.

Tabela 2 - Matriz do delineamento experimental com valores codificados e reais

\begin{tabular}{ccccc}
\hline Ensaios & $\begin{array}{c}\text { Óleo de } \\
\text { Girassol }\end{array}$ & Água & $\begin{array}{c}\text { Óleo de Girassol } \\
(\mathbf{m L})\end{array}$ & Água $(\mathbf{m L})$ \\
\hline $\mathbf{1}$ & -1 & -1 & 12,5 & 12,5 \\
$\mathbf{2}$ & +1 & -1 & 37,5 & 12,5 \\
$\mathbf{3}$ & -1 & +1 & 12,5 & 37,5 \\
$\mathbf{4}$ & +1 & +1 & 37,5 & 37,5 \\
$\mathbf{5}$ & $-1,41$ & 0 & 0 & 25 \\
$\mathbf{6}$ & $+1,41$ & 0 & 50 & 25 \\
$\mathbf{7}$ & 0 & $-1,41$ & 25 & 0 \\
$\mathbf{8}$ & 0 & $+1,41$ & 25 & 50 \\
$\mathbf{9}$ & 0 & 0 & 25 & 25 \\
$\mathbf{1 0}$ & 0 & 0 & 25 & 25 \\
$\mathbf{1 1}$ & 0 & 0 & 25 & 25 \\
\hline
\end{tabular}

\subsection{Processamento dos Pães Tipo Forma}

Os ingredientes foram pesados em balança semi-analítica separadamente. Aplicou-se o método direto, onde todos os ingredientes são colocados simlutaneamente no início da etapa de mistura, com exceção do sal e água. Eles foram misturados em misturadora de escala semiindustrial durante 1 minuto em baixa velocidade para a homogeneização dos ingredientes, em seguida foi adicionada a água e misturada por 3 minutos em velocidade média, por último foi adicionado o sal e a massa foi misturada em alta velocidade por 6 minutos até o seu completo 
desenvolvimento. As massas foram divididas em porções de $250 \mathrm{~g}$ e moldadas na forma de elipses manualmente. Foram imersas nas soluções de óleo de girassol/água correspondentes por 2 minutos, o excesso de solução remanescente na superfície das massas foi retirado por gravidade. Elas foram colocadas em fôrmas de folha galvanizada de ferro de chapa única para pão de forma sem tampa. Em seguida, colocadas em câmara de fermentação regulada a temperatura de $28{ }^{\circ} \mathrm{C} \pm 2{ }^{\circ} \mathrm{C}$ e $80 \%$ de umidade relativa, durante uma hora e trinta minutos. Ao final da fermentação, as massas foram assadas sem vapor durante 20 minutos a temperatura de $220{ }^{\circ} \mathrm{C}$ em forno elétrico de lastro Continental Advance Turbo®. Os pães foram resfriados durante uma hora em temperatura ambiente.

\subsection{Avaliação Física das Massas e dos Pães Tipo Forma}

Avaliação do processo de fermentação pela técnica de perfil livre de expansão: A expansão das massas durante o processo de fermentação foi avaliada através do procedimento experimental de Perfil Livre de Expansão desenvolvido por Gabric et al. (2011), com modificações, onde as massas foram consideradas como elipses truncadas, conforme ilustra a figura 1.

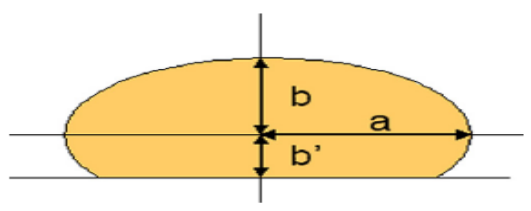

Figura 1 -Parâmetros de elipse truncada

O volume das massas para cada tempo de medição foi calculado com base na fórmula da elipse e sua rotação em torno do eixo y, conforme demonstra a equação 2.

$V\left(c m^{3}\right)=\pi a^{2}\left(\frac{2 b}{3}+b^{\prime}-\frac{b^{3}}{3 b^{2}}\right)$

Onde: $\mathrm{a}=$ largura da elipse $(\mathrm{cm}) ; \mathrm{b}=$ altura da elipse - do centro a elipse superior $(\mathrm{cm})$; $b^{\prime}=$ altura da elipse - do centro para a parte inferior $(\mathrm{cm})$.

O fator de expansão do volume $\left(\mathrm{cm}^{3}\right)$ - VEF, foi calculado através da equação 3 .

$V E F=\frac{V-V_{o}}{V_{o}}$

Onde: $\mathrm{V}=$ Volume da massa ao longo do tempo de fermentação $\left(\mathrm{cm}^{3}\right) ; \mathrm{V}_{\mathrm{o}}=$ Volume incial da massa $\left(\mathrm{cm}^{3}\right)$.

Volume Produzido: Porções de massa de $15 \mathrm{~g}$, foram colocadas em provetas de $100 \mathrm{~mL}$ previamente esterilizadas para medição dos volumes das massas durante o processo de fermentação. Para o cálculo do volume produzido $(\Delta \mathrm{V})$ utilizou-se a subtração entre o volume final produzido e o volume inicial das massas na provetas, os resultados expressos em $\mathrm{mL}$.

Volume Específico: As massas dos pães foram determinadas em balança semi-analítica e expressa em gramas. $\mathrm{O}$ volume deslocado dos pães foi medido através de preenchimento de recipiente plástico com semente de painço, expressando o resultado $\mathrm{mL}$. O volume específico 
foi calculado pela divisão do volume deslocado do pão $(\mathrm{mL})$ pela sua massa $(\mathrm{g})$, segundo método $\mathrm{n}^{\circ}$ 72-10 da AACC (1995).

Densidade: A densidade foi calculada através da relação inversa entre o volume deslocado e o peso da amostra assada.

Índice de Expansão: Para o cálculo do índice de expansão foi adaptada a medotologia utilizada por Silva et al. (2003) para pães de queijo. As massas foram moldadas na forma esférica para permitir as medições do diâmetro e da altura. com auxílio de régua milimetrada simples. O Índice de Expansão (IE) dos pães foi calculado através da equação 4:

Índice de Expansão $(I E)=\frac{\frac{\left(D_{p}+H_{p}\right)}{2}}{\frac{\left(D_{m}+H_{m}\right)}{2}}$

Onde: $\mathrm{D}_{\mathrm{p}}$ e $\mathrm{H}_{\mathrm{p}}=$ Diâmetro e altura dos pães após o forneamento $(\mathrm{cm}) ; \mathrm{D}_{\mathrm{m}}$ e $\mathrm{H}_{\mathrm{m}}=$ Diâmetro e altura das massas moldadas $(\mathrm{cm})$.

\subsection{Análise Estatística}

A avaliação dos resultados dos parâmetros físicos no desenvolvimento das formulações através do delineamento composto central rotacional foi realizada por superfície de resposta e suas respectivas curvas de contorno após a análise de variância (ANOVA) e regressão, foi realizado teste de médias ao nível de $5 \%$ de significância. A análise foi realizada no programa STATISTICA 7.0.

\subsection{RESULTADOS E DISCUSSÃO}

A tabela 3 apresenta os valores obtidos para os parâmetros físicos dos pães tipo forma.

Tabela 3 - Parâmetros físicos dos pães tipo forma

\begin{tabular}{cccccccc}
\hline Ensaios $^{1}$ & $\begin{array}{c}\text { Óleo } \\
(\mathbf{m L})\end{array}$ & $\begin{array}{c}\text { Água } \\
(\mathbf{m L})\end{array}$ & $\begin{array}{c}\text { Volume } \\
\text { Específico } \\
(\mathbf{m L} / \mathbf{g})\end{array}$ & $\begin{array}{c}\text { Densidade } \\
(\mathbf{g} / \mathbf{m L})\end{array}$ & $\begin{array}{c}\text { Índice de } \\
\text { Expansão }\end{array}$ & $\begin{array}{c}\text { Volume } \\
\text { Produzido } \\
(\mathbf{m L})\end{array}$ & $\begin{array}{c}\text { Fator de } \\
\text { expansão } \\
\left(\mathbf{c m}^{\mathbf{3}}\right)\end{array}$ \\
\hline E01 & 12,5 & 12,5 & $3,14^{\mathrm{c}} \pm 0,02$ & $0,31^{\mathrm{d}} \pm 0,01$ & $1,29^{\mathrm{b}} \pm 0,01$ & $21,16 \pm 1,03$ & $1,86 \pm 0,02$ \\
E02 & 37,5 & 12,5 & $3,15^{\mathrm{c}} \pm 0,03$ & $0,31^{\mathrm{d}} \pm 0,01$ & $1,28^{\mathrm{b}} \pm 0,02$ & $17,83 \pm 0,63$ & $1,84 \pm 0,01$ \\
E03 & 12,5 & 37,5 & $2,36^{\mathrm{f}} \pm 0,02$ & $0,43^{\mathrm{a}} \pm 0,01$ & $1,23^{\mathrm{c}} \pm 0,01$ & $9,33 \pm 0,76$ & $1,44 \pm 0,01$ \\
E04 & 37,5 & 37,5 & $2,84^{\mathrm{d}} \pm 0,03$ & $0,37^{\mathrm{c}} \pm 0,01$ & $1,26^{\mathrm{c}} \pm 0,01$ & $13,83 \pm 1,04$ & $1,62 \pm 0,04$ \\
E05 & 0 & 25 & $2,42^{\mathrm{e}} \pm 0,02$ & $0,40^{\mathrm{b}} \pm 0,01$ & $1,18^{\mathrm{d}} \pm 0,01$ & $9,33 \pm 0,76$ & $1,47 \pm 0,03$ \\
E06 & 50 & 25 & $2,33^{\mathrm{e}} \pm 0,02$ & $0,45^{\mathrm{a}} \pm 0,01$ & $1,17^{\mathrm{d}} \pm 0,02$ & $9,83 \pm 1,25$ & $1,39 \pm 0,02$ \\
E07 & 25 & 0 & $3,51^{\mathrm{a}} \pm 0,04$ & $0,27^{\mathrm{e}} \pm 0,01$ & $1,40^{\mathrm{a}} \pm 0,04$ & $23,16 \pm 1,11$ & $2,14 \pm 0,03$ \\
E08 & 25 & 50 & $2,83^{\mathrm{d}} \pm 0,02$ & $0,39^{\mathrm{b}} \pm 0,01$ & $1,27^{\mathrm{b}} \pm 0,02$ & $17,16 \pm 0,76$ & $1,70 \pm 0,01$ \\
E09 & 25 & 25 & $3,34^{\mathrm{b}} \pm 0,02$ & $0,28^{\mathrm{e}} \pm 0,01$ & $1,33^{\mathrm{b}} \pm 0,05$ & $25,00 \pm 1,00$ & $1,97 \pm 0,01$ \\
E10 & 25 & 25 & $3,39^{\mathrm{b}} \pm 0,02$ & $0,28^{\mathrm{e}} \pm 0,01$ & $1,35^{\mathrm{a}} \pm 0,02$ & $25,50 \pm 0,50$ & $2,00 \pm 0,01$ \\
E11 & 25 & 25 & $3,38^{\mathrm{b}} \pm 0,05$ & $0,28^{\mathrm{e}} \pm 0,01$ & $1,37^{\mathrm{a}} \pm 0,02$ & $24,83 \pm 1,44$ & $1,99 \pm 0,04$ \\
\hline
\end{tabular}

O volume específico foi influenciado pela diferentes concentrações da solução de glazeamento, o maior valor foi observado para o ensaio $7(3,51 \mathrm{~mL} / \mathrm{g})$, com solução contendo 
apenas óleo de girassol, quando a solução foi composta de $50 \mathrm{~mL}$ de óleo de girassol e $25 \mathrm{~mL}$ de água, o volume específico obtido foi de $2,33 \mathrm{~mL} / \mathrm{g}$. Não houve diferenças significativas entre os ensaios 9 a 11; 1 e 2; 4 e 8 . Os valores de densidade variaram de $0,27 \mathrm{~g} / \mathrm{mL}$, para o ensaio 7 a $0,45 \mathrm{~g} / \mathrm{mL}$, para o ensaio 6 .

O maior índice de expansão foi apresentado pelo ensaio 7, com 1,40; a solução composta de $50 \mathrm{~mL}$ de óleo de girassol e $25 \mathrm{~mL}$ de água proporcionou a redução deste parâmetro para 1,17; sendo o menor valor obtido. Este comportamento refletiu no volume produzido e no fator de expansão, onde os ensaios correspondentes aos pontos centrais, 9 a 11, obtiveram os maiores valores entre 24,83 e $25,50 \mathrm{~mL}$ de volume produzido e para o fator de expansão máximo, o ensaio $7, \operatorname{com} 2,14 \mathrm{~cm}^{3}$.

A figura 2 apresenta a superfície de resposta e a curva de contorno da ação da solução de glazeamento sobre o fator de expansão máximo das massas.
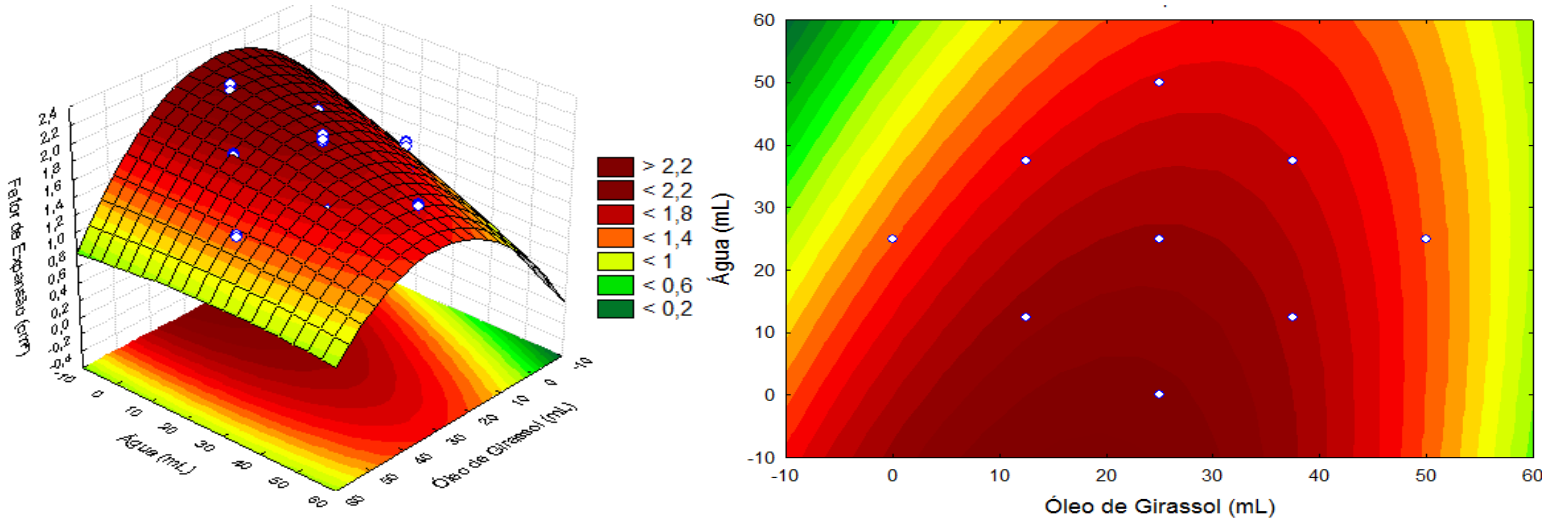

Figura 2 - Superfície de resposta, curva de contorno da ação da solução de glazeamento sobre o fator de expansão máximo da massas

A zona de maximização do fator de expansão máximo das massas ocorreu entre a incorporação de 0 a $35 \mathrm{~mL}$ de óleo de girassol e até $25 \mathrm{~mL}$ de água, para valores superiores a $1,80 \mathrm{~cm}^{3}$, verificou-se que soluções apenas contendo água não são eficazes na melhoria do fator de expansão das massas, isto pode estar relacionado à capacidade de que a solução de glazeamento tem de reter a água durante o processo fermentativo, colaborando para melhor produção de gás pelas leveduras e consequente maior expansão da massa.

A figura 3 apresenta a superfície de resposta e curva de contorno da ação do açaí em pó e quitosana sobre o volume produzido das massas.

A região de maior volume produzido das massas foi obtida entre a utilização de soluções de glazeamento compostas de até $25 \mathrm{~mL}$ de água e até $30 \mathrm{~mL}$ de óleo de girassol, correspondento a volumes produzidos acima de $20 \mathrm{~mL}$. Soluções compostas somente com o óleo e em quantidades acima de $35 \mathrm{~mL}$ reduzem o volume produzido das massas, bem como soluções compostas somente com água produzem volumes inferiores a $20 \mathrm{~mL}$, o uso combinado de água e óleo de girassol mostrou-se eficiente no aumento da capacidade de expansão da massa. Casper et al. (2006) afirma que hidrogéis e outros ingredientes como óleo líquido e água podem conferir barreira à umidade em produtos de panificação, melhorando a sua qualidade, fato que foi observado neste estudo. 

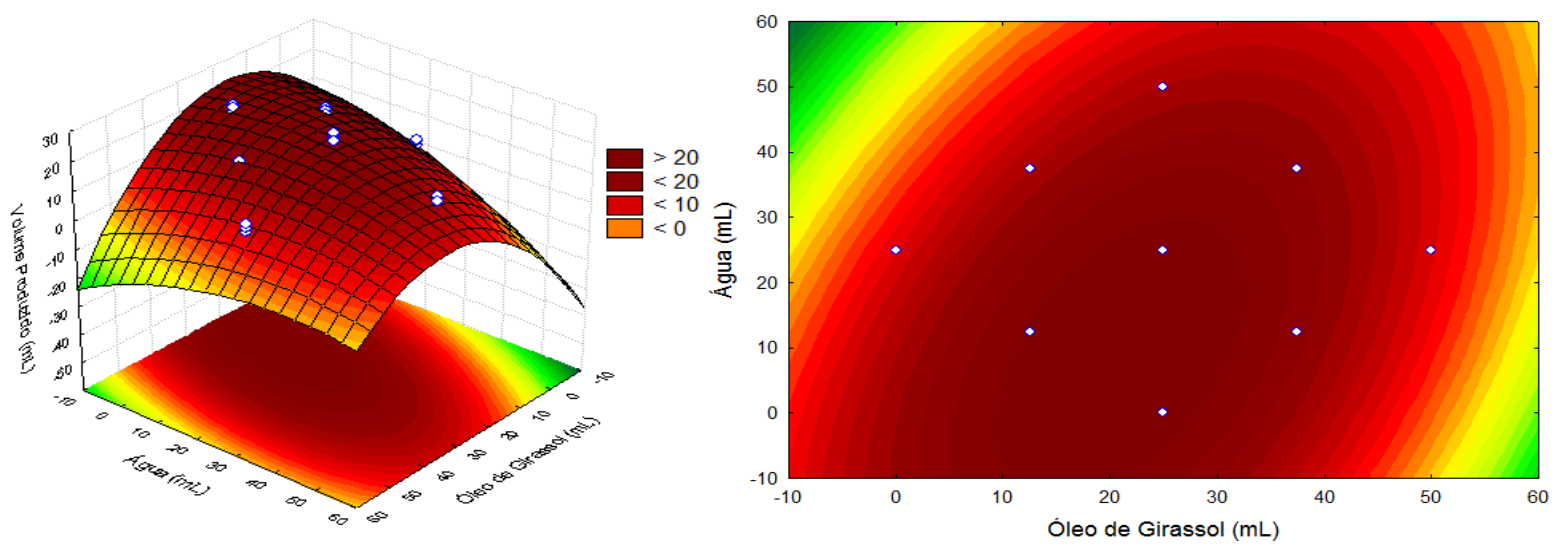

Figura 3 - Superfície de resposta, curva de contorno da ação da solução de glazeamento sobre o volume produzido das massas

A figura 4 fornece a superfície de resposta e curva de contorno da ação da solução de glazemaento no volume específico dos pães.
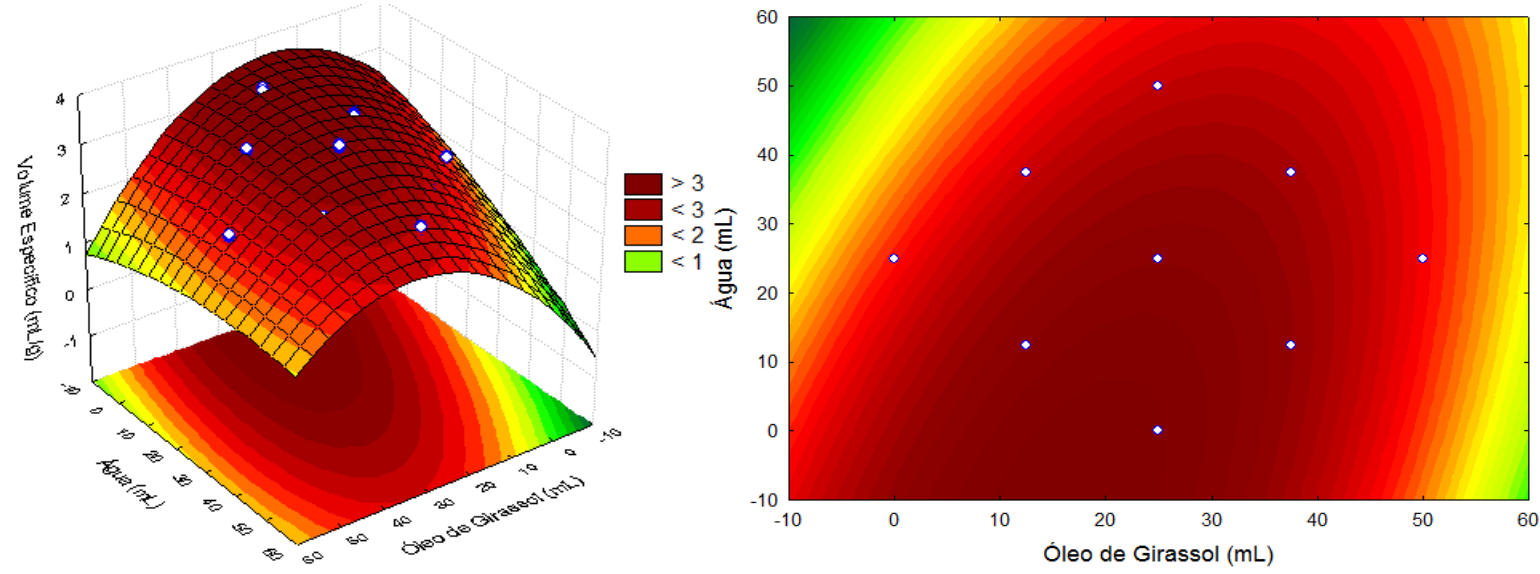

Figura 4 - Superfície de resposta, curva de contorno da ação da solução de glazeamento sobre o volume específico dos pães

O volume específico foi otimizado na região que corresponde às soluções adicionadas de 0 a $15 \mathrm{~mL}$ de água e de 5 a $25 \mathrm{~mL}$ de óleo de girassol, para volumes específicos superiores a 3,00 mL/g. Observou-se que a adição de quantidades superiores a $25 \mathrm{~mL}$ de água produzem volumes específicos inferiores a $2,00 \mathrm{~mL} / \mathrm{g}$, ou seja, pães de qualidade inferior, para o óleo de girassol, isto foi observado apenas para quantidades maiores que $45 \mathrm{~mL}$.

Jahromi et al. (2012) relatam que o uso de soluções de glazeamento, como o óleo de girassol, eleva a viscosidade do sistema alimentício durante o forneamento, produzindo efeito de superfície, o que melhora as características físicas da massa durante o forneamento, elevando o volume específico, o que foi observado neste estudo, onde a incorporação do óleo de girassol em solução de glazeamento elevou o volume específico dos pães em detrimento da redução da densidade.

A figura 5 apresenta a superfície de resposta e a curva de contorno da ação da solução de glazeamento sobre a densidade dos pães tipo forma. 

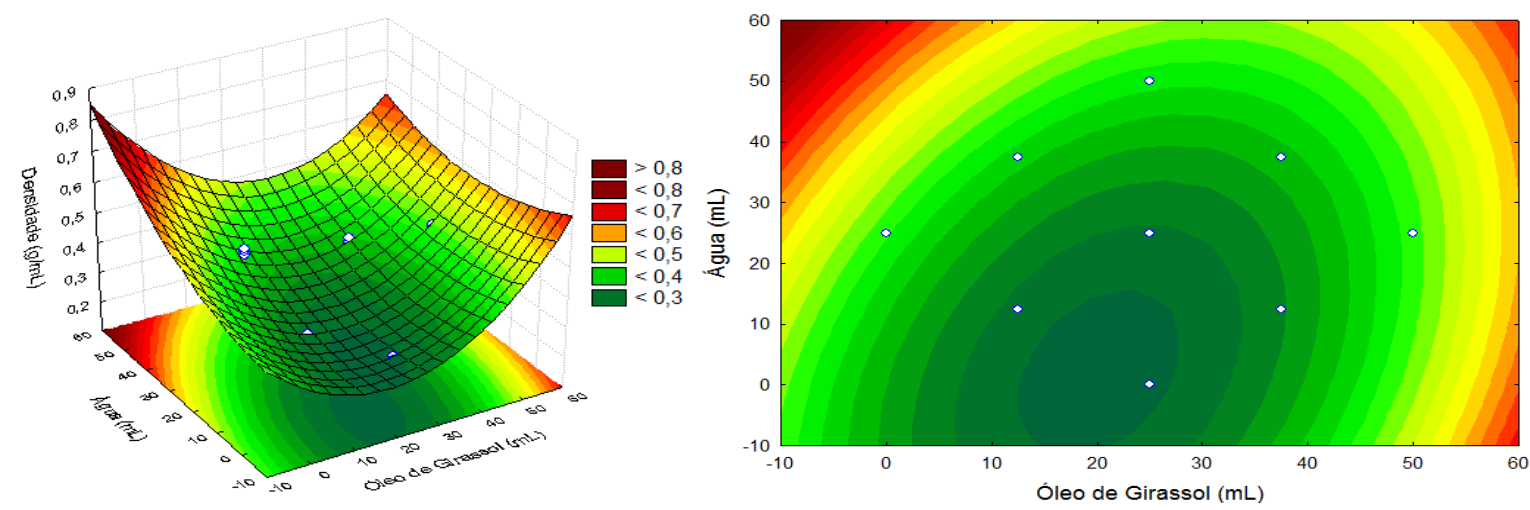

Figura 5 - Superfície de resposta e curva de contorno da ação da solução de glazeamento sobre a densidade dos pães

De acordo com a superfície de resposta obtida, foi observada uma região de mínimo para a densidade, o que é desejável, correspondeu a soluções contendo até $15 \mathrm{~mL}$ de água e entre 15 e $25 \mathrm{~mL}$ de óleo de girassol. Para Esteller e Lannes (2005), pães que possuam alta densidade fornecem características desagradáveis para o consumidor, a determinação das condições ótimas de adição da solução de glazeamento para que se obtenha pães com elevado volume específico e reduzida densidade foi eficaz.

A figura 6 fornece a superfície de resposta e curva de contorno da ação da solução de glazeamento sobre o índice de expansão dos pães.
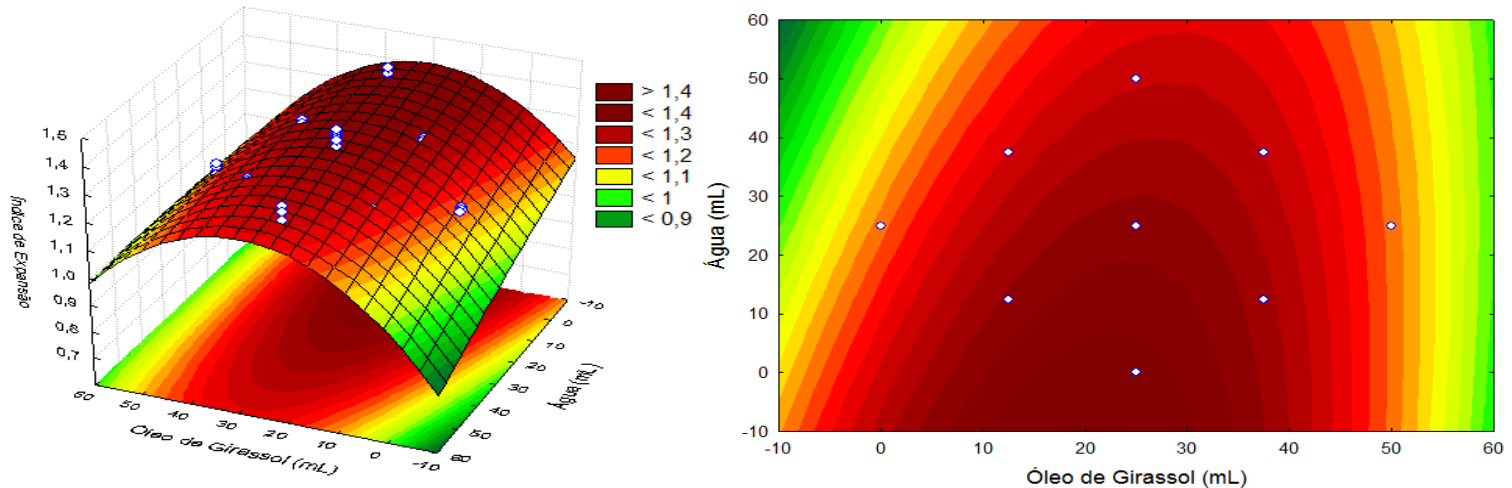

Figura 6 - Superfície de resposta e curva de contorno da ação da solução de glazeamento sobre o índice de expansão dos pães

A maximização do índice de expansão, para valores superiores a 1,30 foi obtida por soluções contendo de até $30 \mathrm{~mL}$ de água e $35 \mathrm{~mL}$ de óleo de girassol. O fenômeno que concerne o uso de soluções de glazeamento e a expansão da massa durante o forneamento também foi observado em estudos desenvolvidos por Jacobson (2003) e Lonergan et al. (2004), houve aumento nas dimensões dos pães, as soluções de glazeamento retardaram a transferência de calor durante o forneamento, havendo maior flexibilidade interna da massa, o que permite maior expansão da massa.

\subsection{CONCLUSÃO}

A utilização de solução de glazeamento composta de óleo de girassol e água mostrou-se eficaz na melhoria dos parâmetros de expansão das massas, a composição da solução que 
otimizou os parâmetros físicos de qualidade é de até $25 \mathrm{~mL}$ de água e $35 \mathrm{~mL}$ de óleo de girassol.

\subsection{REFERÊNCIAS}

AMERICAN ASSOCIATION OF CEREAL CHEMISTS - A. A. C. C. Approved methods of American Association of Cereal Chemists. 9 ed. St. Paul: 1995.

CASPER, J. L.; OPPENHEIMER, A. A.; WEBER, J. L.; ERICKSON, B. J.; RAY, M. M. High expansion dough compositions and methods. United States patent No 0083841, 2006.

CASPER, J. L.; OPENHEIMER, A. A.; ERICKSON, B. Dough compositions having a moisture barrier and related methods. United States patent no 0275128, 2007.

CAUVAIN, S. P.; YOUNG, L. S. Tecnologia da Panificação. Barueri, São Paulo: Ed. Manole, 2009. 418 p.

CHIN, N.L.; ABDU, R. R.; HASHIM, D.M.; KOWNG, S.Y. 2010. Palm oil shortening effects on baking performance of white bread. J. Food Process Eng., v. 33, 413-433, 2010

ESTELLER, M. S.; LANNES, S. C. S. Parâmetros complementares para fixação de identidade e qualidade de produtos panificados. Ciência e Tecnologia de Alimentos, v. $25 \mathrm{n}$. 4, p. 802-806, 2005.

GABRIC, D.; BEN-AISSA, F.; LE-BAIL, A.; MONTEAU, J. Y.; CURIC, D. Impact of process conditions on the structure of pre-fermented frozen dough. J. Food Eng., v.105, p. 361-366, 2011.

JACOBSON, S. M. H. Dough product treatment process and products thereof. United States patent $\mathrm{n}^{\circ}$ 0203091, 2003.

JAHROMI, S. H. R.; YAZDI, F. T.; KARIMI, M.; MORTAZAVI, S. A.; DAVOODI, M. G. F.; POURFARZAD, A.; SOURKI, A. H. Application of glazing for bread quality improvement. Food Biop. Technol, v. 5, p. 2381-2391, 2012.

LONERGAN, D. A.; RODNE, T. R.; WANG, I. A. F. Fried texture imparting glaze for a food intermediate and method of making a product and a product produced thereby. United States patent $\mathrm{n}^{\circ} 0009272,2004$

PRIMO-MARTIN, C.; VAN DE PIJPEKAMP, A.; VANVLIET, T.; DE JONGH, H.H.J.; PLITJTER, J. J.; HAMER, R. J. The role of gluten network in crispness of bread crust. $J$. Cereal Sci. 43, 342-352, 2006.

SILVA, M. R.; GARCIA, G. K. S.; FERREIRA, H. F. Caracterização química, física e avaliação da aceitação de pão de queijo com baixo teor energético. Alim e Nutr, v. 14, n. 1, p. 69-75, 2003. 